\title{
Study of the Fault Diagnosis Model of High Pressure Roller Mill Gearbox Lubrication System
}

\author{
Xijuan Wang ${ }^{*}, 1$, Lei Zhang ${ }^{1}$, Jingxiao Feng ${ }^{2}$, Wei Zhou ${ }^{2}$ and Haiyan Gao ${ }^{2}$ \\ ${ }^{I}$ School of Physical and Electrical Information, Luoyang Normal University, Luoyang 471022, Henan, China \\ ${ }^{2}$ Luoyang Mining Machinery Research Institute, Luoyang 471039, Henan, China
}

\begin{abstract}
The fault phenomenon of high pressure roller mill gearbox lubrication system is not easy to be find in many cases, the fault of system is easy to be ignored, and it is more difficult to judge with the traditional method. For this reason, the fault diagnosis model of the particle swarm neural network has been established by using actual sample data, determining the cause of fault through the actual monitoring data. The practice has proved that it has better prediction effect.
\end{abstract}

Keywords: BP network, fault diagnosis, high pressure roller mill, particle swarm algorithm.

\section{INTRODUCTION}

The high pressure roller mill is widely used in mining, and cement enterprises etc. As an important equipment of crushing, it directly affects the production and benefit of enterprises. It is well received by enterprise because of its characteristics of energy saving and high efficiency, and has been widely promoted. As the important transmission mechanism, the high pressure roller mill gearbox directly affects the normal operation of the equipment, but its lubrication system is the assurance of safe and reliable production. Whether the lubrication system is working or the problem can be found quickly. Whether the cause of the problem is quickly identified for troubleshooting. These questions have become a barrier in the daily inspection and maintenance. But in practical applications, because of limitations of the technical level of the operator, it can not be quickly found when the fault occurs, which leads to equipment damage, due to which the production can't continue. The situation thus exerts great economic losses and is not conducive to the repair and maintenance of the equipment, and resultantly it can bring a lot of trouble for the production of the enterprise. So, finding a fast and effective diagnosis method to identify ahead the fault of lubrication system, so that it can be treated by the maintenance personnel, has thus become a very valuable subject. For this, the paper presented the method of particle swarm neural network fault diagnosis, which is established based on the actual data. The paper has diagnosed and predicted the fault by using the actual data, and the practice showed that prediction result is very good.

\section{PARTICLE SWARM OPTIMIZATION ALGO- RITHM}

\subsection{The Basic Principle of Particle Swarm Optimization Algorithm}

The particle swarm optimization algorithm is the result of the study of birds prey behavior. It is a kind of optimization

\footnotetext{
*Address correspondence to this author at the School of Physical and Electrical Information, Luoyang Normal University, Luoyang 471022, Henan, China; Tel: +8615937912295; E-mail: fjxwxj@126.com
}

tool based on iteration. Firstly, it is initialized to a group of random particles (random solution) when the optimization began, and then it can find the optimal solution through iteration. In each iteration, the particles can update themselves by tracking between two extremes. The first extreme value is the optimal solution currently found in the whole population. The extreme is the global extremumgBest. In addition, we need not the whole population, but only parts as the neighbor of particles, then the extreme in all the neighbors is the local extremum-1Best. The second extreme value is the optimal solution found by the particle itself, which is known as the individual extremum-pBest. The position and speed of random initialization of particles composed of the initial population is calculated by the algorithm. The initial population is evenly distributed in the solution space. The position and speed of the ith particle in the n-dimensional solution space can be respectively represented as $X_{i}=\left(X_{i 1}, X_{i 2}, \cdots, X_{i d}\right)$ and $V_{i}=\left(V_{i 1}, V_{i 2}, \cdots, V_{i d}\right)$, to find the optimal solution through iteration. In each iteration, the particle update occurs at own speed and position by tracking the two extremes. One extreme is the optimal solutions so far found about particle itself, which is called the individual extremum $\left.P=P_{1}, P_{2}, \cdots, P_{i d}\right)$, that is pBest. Another extreme is the optimal solutions so far found about the particles' neighborhood, which is called the optimal particles $P g=|P g 1, P g 2, \cdots, P g d|$ found throughout the neighborhood, that is gBest $[1,2]$. According to the formulas (1) and (2), the particles update occurs at own speed and position:

$$
\begin{aligned}
& v_{i d}^{(t+1)}=w \times v_{i d}^{(t)}+c_{1} \times r_{1} \times\left(p_{i d}^{(t)}-x_{i d}^{(t)}\right) \\
& +c_{2} \times r_{2} \times\left(p_{g d}^{(t)}-x_{i d}^{(t)}\right) \\
& x_{i d}^{(t+1)}=x_{i d}^{(t)}+v_{i d}^{(t)}
\end{aligned}
$$

where: $t$ is the number of iterations; $\omega$ is the inertia weight, its value range is usually have [0.2,1.2]; $r_{1}$ and $r_{2}$ are 
random functions between $[0,1] ; c_{1}$ and $c_{2}$ are acceleration constants, usually value between $[0,2] ; r_{1}$ and $r_{2}$ are two independent random functions, its value range is $[0,1] ; v_{i d}^{t}$ is the speed of particle $\mathrm{i}$ when the iteration time is $\mathrm{t}$; and $x_{i d}^{t}$ is the position of particle $\mathrm{i}$ when the iteration time is $t$.

Inertia weight $\omega$ can improve the convergence performance of the algorithm. The ability of global optimization is strong when $\omega$ becomes large, therefore, it is mainly used in the initial stage of the particle swarm optimization. The ability of local optimization is strong when $\omega$ becomes small, in this case it is mainly used in the later stage of the particle swarm optimization. The transformation formula of $\omega$ is as follows:

$\omega=\omega_{\max }-\frac{\left(\omega_{\max }-\omega_{\min }\right)}{k_{\max }} \times k$

where: $\omega_{\max }$ is the maximum inertia factor; $\omega_{\min }$ is the minimum inertia factor; $k$ is the number of iterations; $k_{\max }$ is the total number of iterations. The fitness particle is as follows:

$$
f_{1}=\frac{1}{\frac{1}{N} \sum_{j=1}^{N}(y-\hat{y})^{2}}
$$

where: $N$ is the number of training samples, $y$ is actual output, and $\hat{y}$ is objective output.

The formula (1) of speed-update mainly consists of three parts. The first part is the previous speed of particles; the second part is the part of "cognitive", it shows the own thinking of the particle; the third part is the part of "society", it shows information sharing and mutual cooperation between the particles.

\subsection{The Realization of Particle Swarm Optimization Algorithm}

The particle swarm optimization algorithm has the characteristics of simple programming and is easy to implement, and the data can achieve good optimization though this algorithm, the flow chart of particle swarm optimization algorithm is shown in Fig. (1).

\section{RBF NEURAL NETWORK}

The RBF neural network is a kind of three-layer-feed forward network. It mainly includes the input layer, the hidden layer and the output layer. RBF neural network has these advantages, such as, simple structure, fast convergence speed, approximate any continuous function with arbitrary precision, etc. The network structure diagram is shown in Fig. (2).

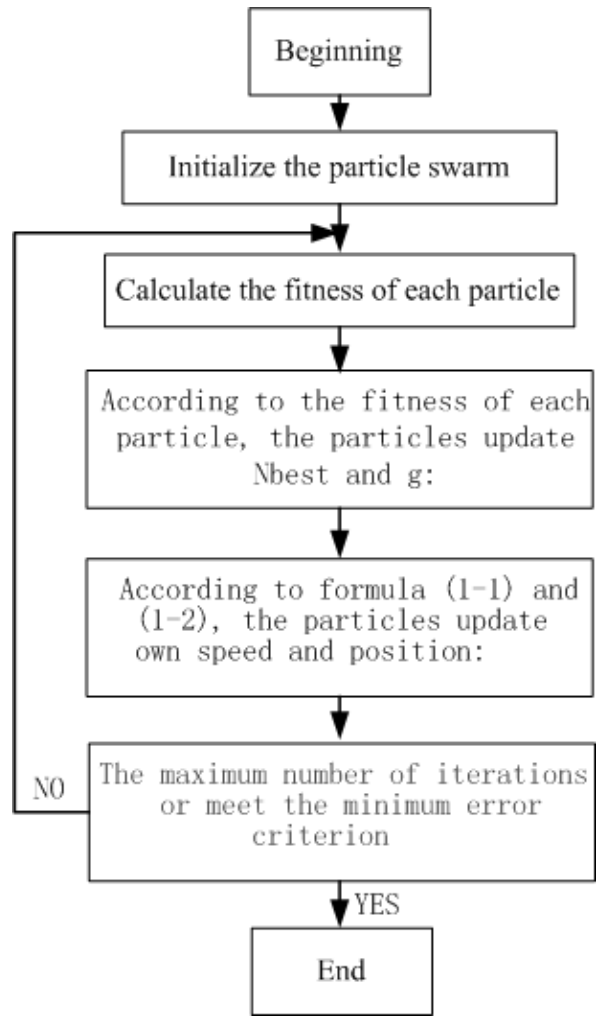

Fig. (1). The flowchart of particle swarm optimization algorithm.

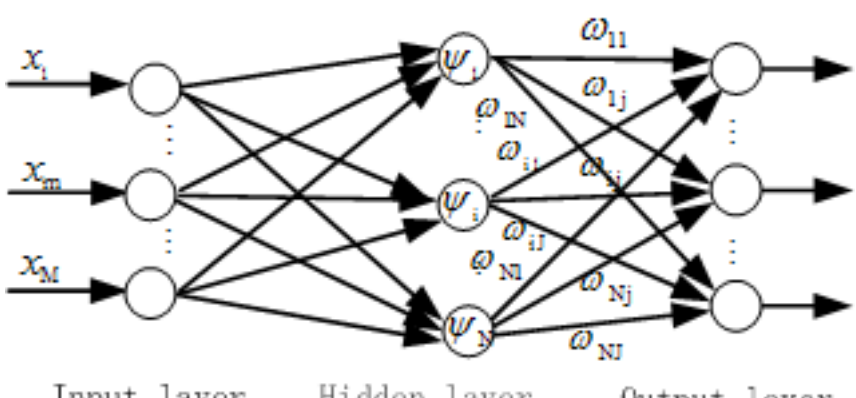

Input layer Hidden layer Output layer

Fig. (2). The RBF neural network structure.

When the training samples of network input are $X_{k}$, the actual output of the $j$-th output neuron is:

$y_{k j}\left(X_{k}\right)=\sum_{i=1}^{N} \omega_{i j} \phi\left(X_{k}, X_{i}\right)$

Generally, "basic function" selected is the Greekn Function; when the Greekn function becomes the Gaussian function:

$$
\begin{aligned}
& \phi\left(X_{k}, X\right)=G\left(X_{k}, X_{i}\right)=G\left(\left\|X_{k}-X_{i}\right\|\right) \\
& =\exp \left(-\frac{1}{2 \sigma_{i}^{2}} \sum_{m=1}^{M}\left(x_{k m}-x_{i m}\right)^{2}\right)
\end{aligned}
$$

The training process of $\mathrm{RBF}$ network is: by the experimental samples to determine the final weight $\omega=\phi^{-1} y$ of training weights $\omega$ between the hidden layer and output layer [3]. 


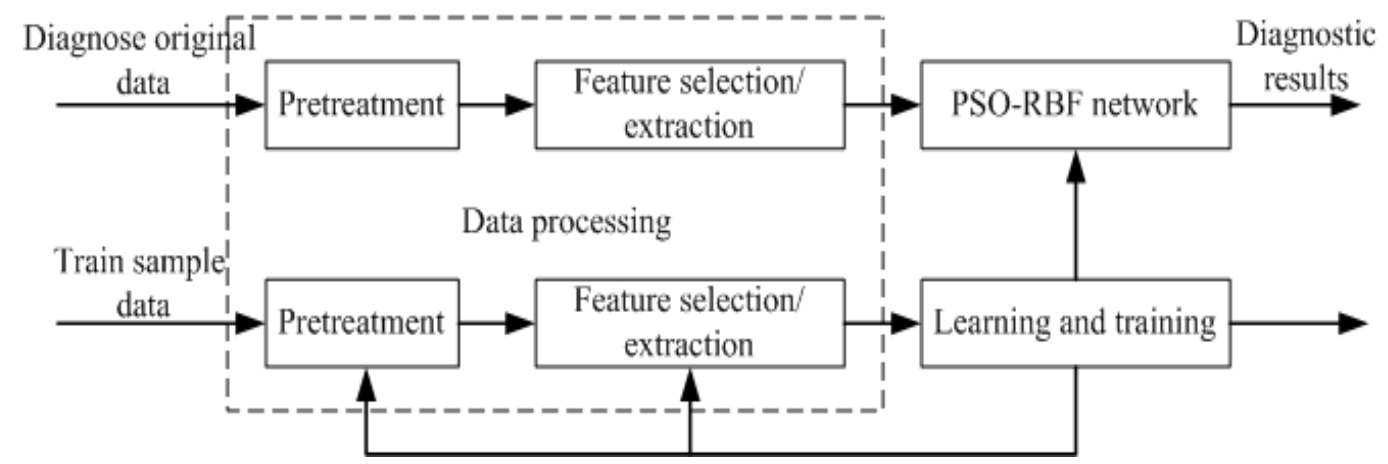

Fig. (3). The structure diagram of PSO-RBF fault diagnosis system.

The fault diagnosis of RBF network has the following steps: The first is the training sample set selection, training the RBF network, determining the structure and parameters of the network, and acquiring the network model of fault diagnosis; The latter verifies the network by using the diagnostic input, and has come up with fault forecast. Before the system learns to train, the training sample data and the real diagnosis data need a pretreatment, so that the network can get more appropriate diagnostic input and training sample. The RBF network is a kind of efficient feed forward neural network, having the best approximation performance and global optimal property, which the other forward network lack. The structure of RBF network is simple, and the training speed is fast, so it is particularly appropriate that the fault identification system of high pressure roller mill reducer is established based on the RBF network [4].

\section{THE NEURAL NETWORK ALGORITHM BASED ON THE OPTIMIZATION OF RADIAL BASIS FUNCTION OF PARTICLE SWARM}

The PSO algorithm is used for the optimization of RBF neural network. Hereinafter referred to as the PSO-RBF network, that is, on the basis of the subtractive clustering algorithm, it gets the number of radial basis center, optimizes the center parameters of RBF through PSO algorithm, adjusts the weights of the network hidden layer to the output layer, and achieves the optimization of the whole RBF neural network.

Algorithm has the following steps:

Collect the training sample, cluster training samples with the subtractive clustering algorithm, obtain the number of RBF centers $h$.

We will take the center value $c_{i}$ of RBF, width $\sigma_{i}$ and the weights of network output layer $w_{i}$ as particle encoding parameters, to conduct real-coding. Initialize PSO and each particle, calculate the adaptive value $f_{i}$ of each particle, compare the adaptive value and obtain BestP and Best $G$.

According to the formulas (1) and (2), obtain the speed $V_{i}$ and position $P_{i}$ of each particle, and at the same time, according to the formula (3), obtain the inertia factor $\omega$.
(4) If the adaptive value achieves requirements or the iterations achieve the maximum iterations, stop the iteration. Otherwise, go to step 2. Repeat it until it achieves the requirements. At this time, by obtaining a group of decoded particles, we can obtain the results of optimization of RBF neural network.

(5) Train the RBF neural network.

\section{THE ESTABLISHMENT OF THE FAULT DIAGNOSIS MODEL OF HIGH PRESSURE ROLLER MILL REDUCER LUBRICATION SYSTEM}

The lubrication system of high pressure roller mill reducer mainly consists of a lubricated and cooled gear box, which can run smoothly and safely. Through the detection of temperature, flow, differential pressure and pressure etc., the system reflects the working condition of an equipment, and gives out the corresponding alarm for fault indication, reminding the technical personnel to overhaul the equipment; the purpose is to ensure the normal operation of the reducer. Although the fault of the equipment indicates the deepseated reason that needs to be judged by the experienced professionals, yet there is a complex nonlinear relationship between fault mode and equipment characteristics, so that the experienced experts even cannot well judge the fault. So, the adaptive learning of neural network and its strong analytical ability toward nonlinear system can solve the problem [5-7]. High pressure roller mill has two groups of gear lubrication systems: moving roller speed reducer and fixed roller speed reducer. They have the same functions and features, have the same structure of the lubrication system, and the detection signals are the same. As an example, the paper establishes the fault diagnosis model with one group of reducer lubrication system.

\subsection{The Determination of Network Model}

According to the operation experience and the actual situation of high pressure roller mill reducer lubrication system, extract the fault samples for its lubrication system by using five operation parameters, sum up five kinds of fault characteristic pattern. Because of the various parameters have different features, the variation range and tendency of these parameters are inequality when the faults occur. And because of the difference of each parameter values is larger, it is adverse to the training and learning of network, so the 
Table 1. The training sample data and the target output.

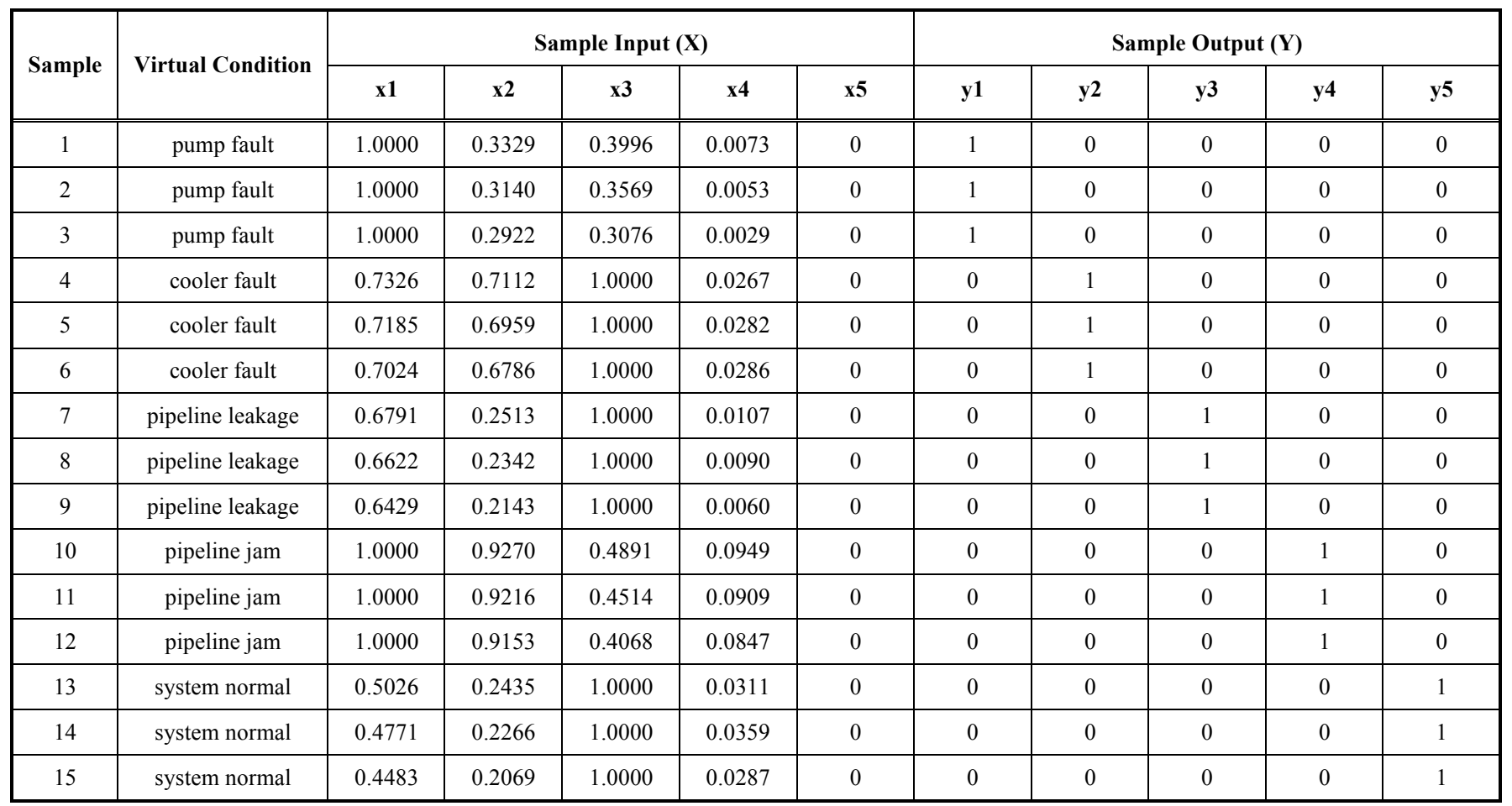

normalization processing of data become the inevitable requirement. And with using particle swarm optimization algorithm, optimize the data, it is concluded that good model data, and has established the model by using RBF network [8-10]. The fault diagnosis model is shown in Fig. (3).

\subsection{The Establishment and Validation of Network Model}

According to the establishment of network model, actual sample data was normalized in order to obtain the sample set of training model, as shown in Table 1. Where: $X$ is the oil temperature of reducer $\left(x_{1}\right)$, the outlet temperature of lubritory $\left(x_{2}\right)$, lubricating oil flow $\left(x_{3}\right)$, lubrication pressure $\left(x_{4}\right)$ and the pressure difference of lubricating oil $\left(x_{5}\right), Y$ is the target output, where: $y_{1}$ is the fault of lubrication pump, $y_{2}$ is the fault of cooler, $y_{3}$ is pipeline leakage, $y_{4}$ is pipeline jam, and $y_{5}$ is system normal. Through the comparison of experiment results, determined by the PSORBF network as follows: the node number of input layer is 5, the node number of output layer is 5 , and the number of RBF center is 15 , initializing the particle population of PSO, gives: $m=40, C_{1}=C_{2}=2$, the range of $\omega$ is $[0.3,0.8]$, the target error $M S E<0.0001$, the maximum iterations is 100 . 1 represents the fault occurrence, and 0 represents that the fault does not occur. The change process of network adaptive value are shown in Fig. (4).

It can be seen from Fig. (4), the selected speed of the adaptive value of PSO-RBF network is fast, its convergence was smooth, and its shock, small. After 60 iterations, it can achieve stable value of -0.1602 , and its convergence effect is good. Five groups of sample data were selected from the actual data acquisition, and had undergone normalized processing, The results shown in Table 2 , verified it by using the model, the diagnostic results were shown in Table $\mathbf{2}$, compared with the concluded that the network model obtained by actual sample data trained had a better classification effect, which is consistent with the actual results shown in Table 3.

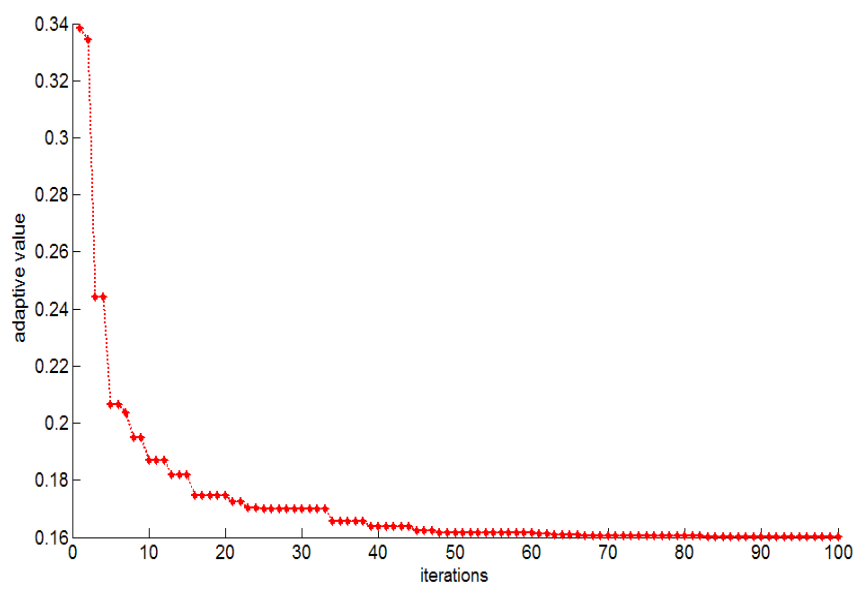

Fig. (4). The change curve of adaptive value.

\section{CONCLUSION}

Based on the actual sample data, the modeling for the fault diagnosis of high pressure roller mill gearbox lubrication system was performed by using particle swarm optimization algorithm and RBF neural network, for the fault classification. The practice proved that the fault prediction of PSO - RBF network model by training on high pressure roller mill reducer lubrication system had good effects. The fault has more accurate classification, so that the technical personnel can quickly find out the cause of the problem, and timely deal with the fault. It can provide a more reliable reference for the overhaul of personnel. 
Table 2. The data and status of actual test sample.

\begin{tabular}{|c|c|c|c|c|c|c|}
\hline Sample & $\mathbf{x 1}$ & $\mathbf{x 2}$ & $\mathbf{x 3}$ & $\mathbf{x 4}$ & $\mathbf{x 5}$ & Virtual Condition \\
\hline \hline 1 & 0.7218 & 0.7056 & 1.0000 & 0.0273 & 0 & cooler fault \\
\hline 2 & 1.0000 & 0.9137 & 0.4356 & 0.0876 & 0 & pipeline jam \\
\hline 3 & 0.6521 & 0.2238 & 1.0000 & 0.0080 & 0 & pipeline leakage \\
\hline 4 & 1.0000 & 0.3028 & 0.3356 & 0.0045 & 0 & lubrication pump fault \\
\hline 5 & 0.4811 & 0.2315 & 1.0000 & 0.0315 & 0 & system normal \\
\hline
\end{tabular}

Table 3. The results of network diagnosis.

\begin{tabular}{|c|c|c|c|c|c|c|c|c|c|c|c|}
\hline \multirow{2}{*}{ Sample } & \multirow{2}{*}{ Diagnosis Results } & \multicolumn{5}{|c|}{ Test Sample Output } & \multicolumn{5}{|c|}{ Sample Output } \\
\hline & & $\mathbf{x 1}$ & $\mathbf{x} 2$ & $\mathbf{x} 3$ & $\mathrm{x} 4$ & $\mathbf{x} 5$ & $\mathbf{y} 1$ & y2 & y3 & y4 & y5 \\
\hline 1 & cooler fault & 0.1053 & 0.9811 & 0.0531 & 0.0326 & -0.0127 & 0 & 1 & 0 & 0 & 0 \\
\hline 2 & pipeline jam & -0.0196 & 0.0132 & 0.0181 & 1.0307 & 0.0098 & 0 & 0 & 0 & 1 & 0 \\
\hline 3 & pipeline leakage & 0.0109 & 0.0137 & 0.9897 & -0.1029 & 0.0117 & 0 & 0 & 1 & 0 & 0 \\
\hline 4 & lubrication pump fault & 1.1019 & 0.0170 & 0.0201 & 0.1051 & 0.0121 & 1 & 0 & 0 & 0 & 0 \\
\hline 5 & system normal & -0.0151 & 0.0191 & 0.0175 & 0.0121 & 0.9781 & 0 & 0 & 0 & 0 & 1 \\
\hline
\end{tabular}

\section{CONFLICT OF INTEREST}

The authors confirm that this article content has no conflict of interest.

\section{ACKNOWLEDGEMENTS}

This study is supported by Science and Technology Research Projects in Henan Province of China (142102210474) and Key Education Department Science and Technology Research Project in Henan Province of China (14A413007).

\section{REFERENCES}

[1] Y. Cao, "Analog Circuit Fault Diagnosis Based on Particle Swarm Optimization," Changchun University of Science and Technology, China, 2005.

[2] H. Yi, "A Study of Support Vector Machines Based Fault Diagnosis and Its Applications," Nanjing University of Aeronautics and Astronautics The Graduate School, China, 2014.
[3] H. Cui, L. Zhang, R. Kang, and X. Lan, "Research on fault diagnosis for reciprocating compressor valve using information entropy and SVM method," Journal of Loss Prevention in the Process Industries, vol. 22, no. 6, pp. 864-967, 2009.

[4] S. X. Ding, P. Zhang, A. Naik, E.L. Dingb, and B. Huang, "Subspace method aided data-driven design of fault detection and isolation systems," Journal of Process Control, vol. 19, no. 9, pp. 1496-1510, 2009.

[5] M. Kano, and Y. Nakagawa, "Data-based process monitoring, process control, and quality improvement: Recent developments and applications in steel industry," Computers \& Chemical Engineering, vol. 32, pp. 12-24, 2008.

[6] B. Huang, H. Ding, X. Zhang, and H. Heng, "Predication method of complex equipent based on RBF neural network," Computer Simulation, no. 1, pp. 14-16, 2014.

[7] L. He, "Research of Attribute Reduction and Engine Fault Diagnosis Based on Rough Set," Southwest Jiaotong University, China.

[8] T. Shu, J. Lan, and Y. Li, "Network traffic prediction with radial basis function neural network based on quantum adaptive particale swarm optimization," Journal of Electronics \& Infornation Technology, no. 9, pp. 2220-2226, 2013.

[9] D. Li, "Research and Development of Fault Diagnosis Expert System Based on Rough Set - Neural Network of Hydraulic Press," Hefei University of Technology, China, 2012.

[10] S. Gong, "The Research on SVM Fault Diagnosis of Mine Hoist," Liaoning Technical University, China, 2013. 